\title{
Use of a Continuous Wave Laser and Pockels Cell for Sensitive High-Resolution Collinear Resonance Ionization Spectroscopy
}

\author{
R. P. de Groote, ${ }^{1,}{ }^{*}$ I. Budinčević, ${ }^{1}$ J. Billowes, ${ }^{2}$ M. L. Bissell, ${ }^{1,2}$ T. E. Cocolios, ${ }^{2}$ G. J. Farooq-Smith, ${ }^{2}$ V. N. Fedosseev, ${ }^{3}$ \\ K. T. Flanagan, ${ }^{2}$ S. Franchoo, ${ }^{4}$ R. F. Garcia Ruiz, ${ }^{1}$ H. Heylen, ${ }^{1}$ R. Li, ${ }^{4}$ K. M. Lynch,${ }^{1,2,5}$ B. A. Marsh, ${ }^{3}$ G. Neyens, ${ }^{1}$ \\ R. E. Rossel, ${ }^{3,6}$ S. Rothe, ${ }^{3}$ H. H. Stroke, ${ }^{7}$ K. D. A. Wendt, ${ }^{6}$ S. G. Wilkins, ${ }^{2}$ and X. Yang ${ }^{1}$ \\ ${ }^{1}$ KU Leuven, Instituut voor Kern-en Stralingsfysica, B-3001 Leuven, Belgium \\ ${ }^{2}$ School of Physics and Astronomy, The University of Manchester, Manchester M13 9PL, United Kingdom \\ ${ }^{3}$ Engineering Department, CERN, CH-1211 Geneva 23, Switzerland \\ ${ }^{4}$ Institut de Physique Nucléaire d'Orsay, F-91406 Orsay, France \\ ${ }^{5}$ Physics Department, CERN, CH-1211 Geneva 23, Switzerland \\ ${ }^{6}$ Institut für Physik, Johannes Gutenberg-Universität, D-55128 Mainz, Germany \\ ${ }^{7}$ Department of Physics, New York University, New York, New York 10003, USA
}

(Received 24 June 2015; published 24 September 2015)

\begin{abstract}
New technical developments have led to a 2 orders of magnitude improvement of the resolution of the collinear resonance ionization spectroscopy (CRIS) experiment at ISOLDE, CERN, without sacrificing the high efficiency of the CRIS technique. Experimental linewidths of 20(1) MHz were obtained on radioactive beams of francium, allowing us for the first time to determine the electric quadrupole moment of the short lived $\left[t_{1 / 2}=22.0(5) \mathrm{ms}\right]{ }^{219} \mathrm{Fr} Q_{s}=-1.21(2) \mathrm{eb}$, which would not have been possible without the advantages offered by the new method. This method relies on a continuous-wave laser and an external Pockels cell to produce narrow-band light pulses, required to reach the high resolution in two-step resonance ionization. Exotic nuclei produced at rates of a few hundred ions/s can now be studied with high resolution, allowing detailed studies of the anchor points for nuclear theories.
\end{abstract}

DOI: 10.1103/PhysRevLett.115.132501

PACS numbers: 21.10.Ky, 21.10.Ft, 21.10.Hw, 27.80.+w

Laser spectroscopy of radioactive ion beams provides key nuclear observables to understand the evolution of the quantum many-body problem at the extremes of isospin by providing model-independent measurements of essential quantum observables. These observables are required to refine the current theories of the atomic nucleus and to further our understanding of the nuclear forces (see, e.g., [1-3]). Alternatively, laser spectroscopy on exotic isotopes also provides input for precision tests of many-body QED [4] or to investigate hyperfine anomalies [5].

As the nucleon drip lines are approached, nuclei often have short half-lives and are produced in minute quantities, which imposes strong efficiency requirements on laser spectroscopy techniques. At the same time, the spectral resolution of these experiments needs to be sufficiently high to allow extraction of all of the observables of interest. Over the past decades, many techniques have been developed with the aim of meeting both requirements, see, for example, [6] for a recent review.

One of these techniques is collinear laser spectroscopy, applied in a number of experiments around the world [7-11]. In this technique an accelerated ion or atom beam

Published by the American Physical Society under the terms of the Creative Commons Attribution 3.0 License. Further distribution of this work must maintain attribution to the author(s) and the published article's title, journal citation, and DOI. of a particular isotope is collinearly overlapped with a continuous wave (cw) laser beam, inducing resonant excitation of the hyperfine levels of the studied isotope. In most of these experiments the hyperfine structure is observed by measuring the fluorescence photons emitted by the deexcitations from the resonantly excited hyperfine levels. The use of accelerated ion beams in a collinear geometry enables typical collinear laser spectroscopy experiments to achieve resonance peak linewidths of the order of $40-70 \mathrm{MHz}[7,12,13]$, which are sufficient to resolve the hyperfine structure in most elements. In combination with a bunched ion beam produced with a linear Paul trap, the technique is nowadays routinely performed on radioactive species with production rates down to several 1000 particles/s [7,8]. A second very successful laser spectroscopic technique to study exotic isotopes is in-source laser spectroscopy [14]. It is based on the efficient resonant excitation of the hyperfine levels using a narrow-band pulsed laser and the subsequent (resonant or nonresonant) laser ionization of these radioisotopes in the ion source. Ion detection is very efficient due to the high quantum efficiency of the particle detector and complete solid angle coverage. This allows very exotic nuclei with production rates down to a few particles per second to be accessed [15]. Resonance ionization spectroscopy (RIS) of radioactive beams is usually only performed with pulsed lasers, since only pulsed lasers 
can provide the high laser power densities required for efficient ionization in an ion source. Because the resonance ionization takes place in a hot cavity, Doppler broadening limits the experimental linewidths to typically $4-5 \mathrm{GHz}$ $[15,16]$. A modification of the in-source approach relies on resonance ionization of atoms in a gas cell [17]. A combination of Doppler and pressure broadening results in linewidths of a few $\mathrm{GHz}$. The in-gas cell laser spectroscopy method has recently been improved by doing the RIS in a supersonic gas jet rather than in the gas cell, which allows for a reduction of the total linewidth to several hundred $\mathrm{MHz}$ [18].

The collinear resonance ionization spectroscopy (CRIS) technique was proposed as a way to combine the efficiency of RIS with the Doppler-free conditions of a collinear experiment [19]. The method relies on collinearly overlapping an atom beam with a laser beam, thus avoiding Doppler and pressure broadening, and uses the detection of the laser-ionized atoms rather than the fluorescence photons, enhancing the sensitivity and selectivity. The first demonstration of the technique in the nineties served as a promising proof of principle, reaching a total efficiency of $10^{-5}$, background rate of $10^{-8}$, and a linewidth of $50 \mathrm{MHz}$ [20]. Results obtained at the dedicated CRIS beam line at ISOLDE, CERN in 2012 demonstrated an improved total experimental efficiency reaching $1 \%$, using a combination of bunched beams and pulsed lasers [21]. This efficiency extended laser spectroscopy measurements to radioactive species with production yields down to 100 particles/s. The spectral linewidth of $1.5 \mathrm{GHz}$ was completely determined by the pulsed Ti-sapphire laser system that was used [22].

Pulsed laser light with a linewidth of the order of $50 \mathrm{MHz}$ has been produced by pulse dye amplification (PDA) of a cw laser beam [20]. With this method, the final linewidth of the pulsed light is limited by the width of the Fourier transform of the laser pulse and further increased by frequency chirp caused by nanosecond scale changes in the refractive index of the medium. For solid-state Ti:sapphire lasers, this chirp can be corrected to reduce the linewidth to $6 \mathrm{MHz}$ [23-26]. Another way of producing spectrally narrow laser pulses is by using injection seeding [27]. An important advantage of both methods is the high output powers such systems can achieve, although both come with the disadvantage of a rather challenging experimental setup. A different approach to the production of narrowband laser pulses uses an electro-optical modulator (EOM) to modulate the amplitude of a cw laser beam rather than amplifying it $[9,28]$. The extinction ratio, defined as the laser power during the pulse divided by the laser power during the off time can be as good as 1:180 [28]. Since there is no amplification of the cw beam, the peak fluence of the laser pulses is low, but the final spectral linewidth is purely determined by the transform of the modulation and is not influenced by frequency chirp effects. An additional advantage is the smaller experimental footprint, since no pump laser or stabilization electronics are required. The radio-frequency modulation can induce sidebands in the atomic spectra which have to be accounted for.

In this Letter, we present the development of a laser ionization method based on a Pockels cell and a cw laser system to overcome some of the disadvantages of the methods described above. Using this system, the spectral resolution of the CRIS experiment was improved by nearly 2 orders of magnitude, without inducing optical pumping to dark states or reducing the experimental efficiency. The spectral linewidth of the laser pulses is purely Fourier limited. The ability to tune the pulse length to the decay time of the atomic excited state under investigation is a key advantage compared to PDA or injection seeding, where the pulse length is fixed. The system was running at a repetition rate of 100 or $200 \mathrm{~Hz}$ to match the Nd:YAG laser, which means the induced sidebands will appear at 100 or $200 \mathrm{~Hz}$. These sidebands are therefore too close to influence the atomic spectra. Furthermore, for a sufficiently long-lived excited state, the ionization laser can be delayed until after the excitation pulse, which has two important implications. First, the excitation laser can probe the atomic system without the perturbation of the strong ionizing laser field, removing potential line shape distortions. Second, power broadening due to both the excitation and the ionization laser is removed completely, even for high laser powers [29-31]. By exciting to a long-lived state, the resolution of the experiment is also enhanced significantly due to the smaller natural linewidth. This advantage is more difficult to exploit for techniques that use fluorescence detection, since the use of weak transitions will considerably reduce signal rates.

The advantages described in the previous paragraph in combination with the efficiency of the CRIS technique will give access to nuclei with lower production rates and shorter half-lives across the nuclear chart. The improved performance of the CRIS technique is illustrated here with the measurement of the electric quadrupole moments of ${ }^{219} \mathrm{Fr}$. This isotope is situated near the region of reflection asymmetry [32-35]. Previous laser spectroscopy experiments performed on francium beams [36-39] did not have the required combination of resolution and sensitivity to extract the electric quadrupole moment of ${ }^{219} \mathrm{Fr}$ $\left(t_{1 / 2}=22.0(5) \mathrm{ms}\right.$ [40]), produced with rates around $10^{3}-10^{4}$ particles/s. From the measured spins and magnetic moments of ${ }^{219,221} \operatorname{Fr}[36,38]$ it was concluded that the unpaired valence proton in these nuclei occupies the $h_{9 / 2}$ orbital, while the charge radii of the francium isotopes follow the trend of the neighboring radium isotopes, indirectly showing that ${ }^{219} \mathrm{Fr}$ is located at the border of a region of octupole collectivity [38]. Direct measurements of octupole moments of radioactive beams with laser spectroscopy require a resolution below $10 \mathrm{MHz}$ and are at present not possible, but indirect information can be derived from the quadrupole collectivity. 
The ${ }^{219,221} \mathrm{Fr}$ beams are produced at the ISOLDE facility at CERN through a spallation reaction in a thick $\mathrm{UC}_{x}$ target induced by $1.4 \mathrm{GeV}$ protons. The francium atoms are surface ionized in a hot capillary tube, accelerated to $40 \mathrm{keV}$, mass separated, and then cooled and bunched in a gas-filled linear Paul trap [41]. The ion beam is then transported into the CRIS experiment [42], where it is neutralized through collisions with a potassium vapor. After passing through a differential pumping section, the neutral fraction of the beam is temporally and spatially overlapped with the laser beams in a UHV interaction region. The time synchronization is controlled using a Quantum Composers QC9258 digital delay generator. Once in the interaction region, the isotopes are resonantly excited through the $7 s^{2} S_{1 / 2}-8 p^{2} P_{3 / 2}$ transition at $422.7 \mathrm{~nm}$ using chopped $\mathrm{cw}$ light. The lifetime of the excited state is $\tau=83.5 \pm 1.5 \mathrm{~ns}$ [43] and very well suited for delayed RIS. The resonantly excited atoms are nonresonantly ionized using $1064 \mathrm{~nm}$ laser light, using different delay times between the excitation and ionization laser pulses. These ions are deflected onto a copper plate, and the secondary electrons are detected using a microchannel plate (MCP). The signals produced by the MCP are then amplified, discriminated, and processed by a National Instruments USB-6211 DAQ card. This USB card is triggered by the Quantum composer every $100 \mathrm{~ms}$, in synchronization with the laser system and ion beam delivery.

The laser system used for the high-resolution resonance laser ionization is presented in Fig. 1. The laser light for the resonant step was provided by a Matisse TS cw Ti-sapphire laser, which was frequency scanned using the Matisse Commander software. A small pickoff of the $2.7 \mathrm{~W}$ fundamental laser beam was sent into a HighFinesse WSU2 wave meter, which was regularly calibrated to an external temperature stabilized HeNe laser. The fundamental laser beam of the Matisse was frequency doubled to $422.7 \mathrm{~nm}$ using a Wavetrain external cavity frequency doubler. The

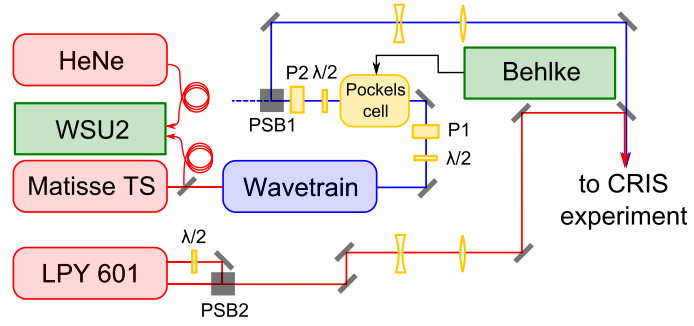

FIG. 1 (color online). A schematic overview of the laser setup. The laser light for the excitation step is produced by a Matisse TS Ti-sapphire continuous wave laser which is frequency doubled by a Wavetrain doubler and sent through a light chopping setup (see text for more details). The $1064 \mathrm{~nm}$ light for the nonresonant ionization step is produced by a Litron LPY 601 50-100 PIV Nd:YAG laser operating at either 100 or $200 \mathrm{~Hz}$. Light of both lasers is focused and spatially overlapped before being sent to the CRIS experiment. laser beam then passed through a half-wave plate, GlanTaylor polarizer $(P 1)$ and Pockels cell, and then through another half-wave plate and a second Glan-Taylor polarizer $(P 2)$. A polarizing beam splitter cube (PSB1) positioned further along the beam path only reflected light with one particular polarization, which was then further transported into the CRIS beam line. The polarization axis of the light was rotated by $\pi / 2$ by the Pockels cell for an applied voltage of $2.4 \mathrm{kV}$. Through the use of a Behlke FSWP91-01 fast square wave pulser, the voltage applied to the Pockels cell could be rapidly switched from 0 to $2.4 \mathrm{kV}$. The combination of the rapid switching of the laser light polarization and the polarization-sensitive beam splitter cube therefore allowed for creating short pulses of laser light. Optimized transmission efficiencies of $65 \%$ and extinction ratios of 1:2000 were achieved. The measured rise and fall times of the light pulses were $10 \mathrm{~ns}$. The power of the $422.7 \mathrm{~nm}$ light was $25 \mathrm{~mW}$ just before the entry window to the CRIS beam line. The laser efficiency obtained with this laser system was comparable to the low-resolution laser system that was used for the previous CRIS experiment [21]. This was determined by comparing back-to-back measurements using the high-resolution laser system and the previous system on ${ }^{206} \mathrm{Fr}$. Figure 2 shows the saturation curve for the excitation step. The red curve is the best fitting saturation function, defined as

$$
I(P)=A \frac{P / P_{0}}{1+P / P_{0}},
$$

which yields a saturation power of $P_{0}=9 \pm 4 \mathrm{~mW}$. Light from the two Nd:YAG cavities in the Litron LPY 601 50-100 PIV laser system was overlapped using a halfwave plate and a polarizing beam splitting cube (PSB2), yielding $2 \times 4 \mathrm{~W}$ of laser light just before the CRIS entry window. The synchronization of the two cavities can be tuned to change the repetition rate of the Litron laser system to either 100 or $200 \mathrm{~Hz}$.

By performing systematic measurements of the hyperfine structure of ${ }^{221} \mathrm{Fr}$, optimal pulse lengths of $100 \mathrm{~ns}$ and

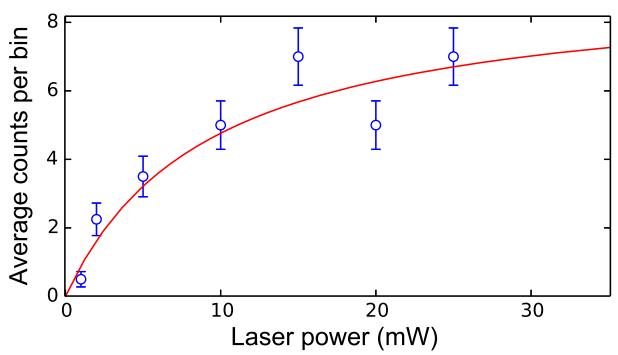

FIG. 2 (color online). Saturation curve for the excitation laser step, obtained by fixing the laser frequency at the resonance frequency of the strongest peak in the left multiplet of ${ }^{221} \mathrm{Fr}$. The ionization laser fired at the end of the excitation pulse, with a power of $8 \mathrm{~W}$. From the best fitting saturation function (shown in red), a saturation power of $P_{0}=9 \pm 4 \mathrm{~mW}$ is extracted. 


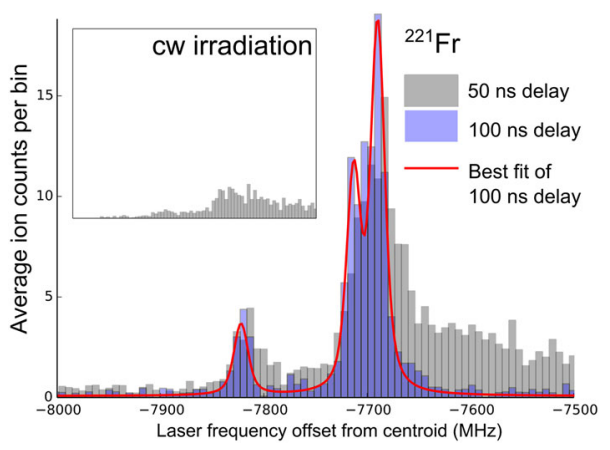

FIG. 3 (color online). Inset: Resonance ionization spectrum with continuous wave laser light in the first step. The scale and range on the $x$ and $y$ axes are the same as the main plot. Main: RIS of the lowest frequency multiplet of ${ }^{221}$ Fr using chopped $\mathrm{cw}$ light with a pulse length of 100 ns for two delay times of the ionization step.

ionization laser delay of $100 \mathrm{~ns}$ with respect to the rise time of the excitation pulse were determined. With these settings the blue histogram in Fig. 3 is obtained. If the ionization step is delayed less then $100 \mathrm{~ns}$, and therefore arrives temporally overlapped with the excitation pulse, the grey spectrum in Fig. 3 is obtained. The peaks broaden and shift to the high-frequency side, and an additional structure also appears on the high-frequency side of the resonance peaks. When a constant $2.4 \mathrm{kV}$ voltage is applied (and the atoms are therefore continuously irradiated), the spectrum in the inset of Fig. 3 is obtained. The event rate is much lower due to optical pumping effects, and the peaks are significantly broadened and shifted to higher frequencies. Figure 3 also shows the best fitting function for the optimal settings (in red), consisting of the sum of Voigt profiles centered on the resonance positions. A total linewidth of $20(1) \mathrm{MHz}$ is obtained. The total linewidth is dominated by the remaining Doppler spread of the atom beam and the spectral broadening of the excitation pulse due to the chopping, estimated at approximately $10 \mathrm{MHz}$ by taking the Fourier transform of the time profile of the laser pulse.

An example of a resonance ionization spectrum of ${ }^{219} \mathrm{Fr}$ is shown in Fig. 4. The linewidth of 20(1) MHz is sufficient to resolve all six expected resonance peaks, which are fitted as described in, e.g., Ref. [38]. The weighted mean of the fitted hyperfine parameters are summarized in Table I. From these parameters, the nuclear magnetic dipole moment $\mu$ and electric quadrupole moment $Q_{s}$ were determined relative to the values for ${ }^{221} \mathrm{Fr}$ in Refs. [36,37]. The newly measured value of $Q_{s}=$ $-1.21(2)$ eb $\left(\beta_{2}=0.094\right)$ for ${ }^{219} \mathrm{Fr}$ is plotted along with the $Q_{s}$ values of other francium isotopes from the literature $[9,36]$ in Fig. 5. The $Q_{s}$ values illustrate the different structures in these odd- $A$ francium isotopes: for $A<215$ the quadrupole moment is nearly zero, as expected in the shell model for isotopes with a half-filled $h_{9 / 2}$ proton orbit [44]. The small deviation from zero is a signature of
TABLE I. Summary of the extracted hyperfine structure constants for the $7 s^{2} S_{1 / 2}$ atomic ground state and $8 p^{2} P_{3 / 2}$ excited state of ${ }^{219,221} \mathrm{Fr}$ (values in MHz). The extracted magnetic dipole moment and electric quadrupole moment are also given. Literature values were obtained from Refs. [36-38].

\begin{tabular}{|c|c|c|c|c|c|}
\hline & $\mathrm{A}\left(7 s^{2} S_{1 / 2}\right)$ & $\mathrm{A}(8 p$ & $\left.P_{3 / 2}\right)$ & $\mu\left(\mu_{N}\right)$ & $Q_{s}(\mathrm{eb})$ \\
\hline & +6 & & & + & \\
\hline & & & & & \\
\hline & & & -8 & & - \\
\hline $21_{\exp }$ & $+6209(1)$ & $+22.3(5)$ & $-87(2)$ & $+1.57(2)$ & $-1.02(3)$ \\
\hline
\end{tabular}

configuration mixing and an increasing contribution from core polarization towards the neutron-deficient region. The large absolute values for the quadrupole moments of ${ }^{219-225} \mathrm{Fr}$ are a signature for deformation in their ground states. The sudden change from strongly negative to strongly positive spectroscopic quadrupole moments is understood in the Nilsson model as due to the changing influence of Coriolis mixing on these prolate deformed structures. Coriolis mixing is strongest in $K=1 / 2$ bands and tends to decouple the odd-particle spin from the deformation axis $[45,46]$. The $9 / 2^{-}$ground state in ${ }^{219} \mathrm{Fr}$ is known to be a member of the $K=1 / 2^{-}$band [47]. Thus the odd-proton spin is decoupled from the nuclear deformation axis [see (b) in Fig. 5], yielding a negative quadrupole moment $(K<I)$. In the heavier francium isotopes, the deformation gradually increases and the nuclear spin gets gradually more coupled to the deformation axis. The $5 / 2^{-}$ ground state in ${ }^{221} \mathrm{Fr}$ was interpreted also as a member of the $K=1 / 2^{-}$band, but with a much smaller decoupling parameter $a$. In ${ }^{223,225} \mathrm{Fr}$ the $3 / 2^{-}$ground state is a member of the $K=3 / 2^{-}$band and thus strongly coupled to the deformation axis $(K=I)$, resulting in a positive quadrupole moment.

In summary, we have presented a novel high resolution, highly efficient collinear resonance ionization spectroscopy technique. By probing a weak atomic transition in francium, a linewidth of 20(1) $\mathrm{MHz}$ was achieved, which represents an improvement of the experimental resolution by nearly 2 orders of magnitude compared to our previous measurements [21]. The advantages of the new method in terms of resolution, efficiency, and systematic line shape

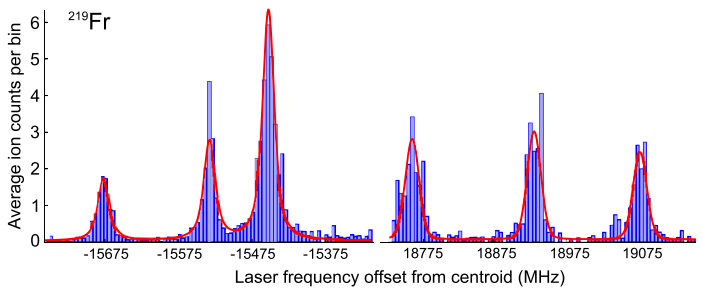

FIG. 4 (color online). Example of an experimental hyperfine spectrum of ${ }^{219} \mathrm{Fr}$ and the best fitting function (red line) consisting of a sum of Voigt profiles centered on the resonance positions. 


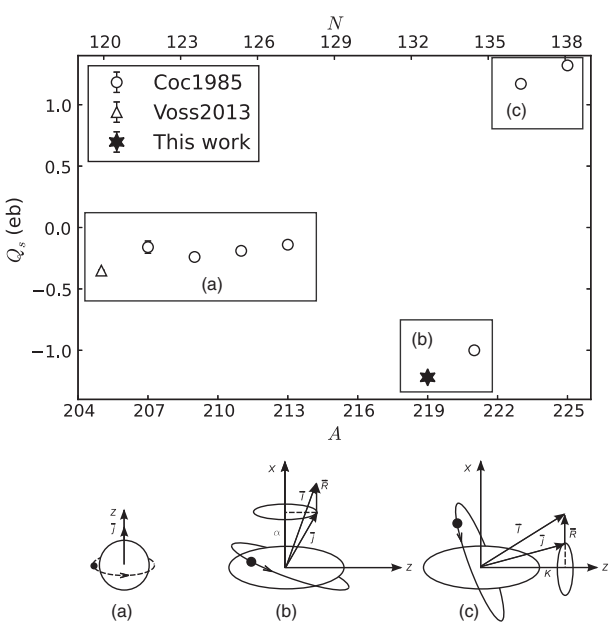

FIG. 5. Spectroscopic quadrupole moment values of ${ }^{219} \mathrm{Fr}$ measured in this work, along with literature values for the neighboring odd francium isotopes. The isotopes on the main figure are grouped and labeled (a),(b) and (c) according to their structure (see also the labeled diagrams below the figure). The $z$ axis in the three schemes is the axis of deformation. $\vec{j}$ is the single particle angular momentum of the valence proton, $\vec{I}$ the total nuclear spin, and $\vec{R}$ the rotational angular momentum of the nucleus. $K$ is the projection of $j$ on $z$ and $\alpha$ is the projection on the $x$ axis.

effects were illustrated by performing laser spectroscopy on beams of radioactive francium, using the $422.7 \mathrm{~nm}$ transition to the excited $8 p^{2} P_{3 / 2}$ state. The performance of the technique allowed measurement of the electric quadrupole moment of ${ }^{219} \mathrm{Fr}\left[t_{1 / 2}=22.0(5) \mathrm{ms}\right]$ for the first time.

We acknowledge the support of the ISOLDE Collaboration and technical teams. We are grateful to the COLLAPS Collaboration for the use of their cw Ti:sapphire laser system and wavetrain doubling unit. This work was supported by the BriX Research Program No. P7/12 and FWO-Vlaanderen (Belgium) and GOA 10/ 010 from KU Leuven, the Science and Technology Facilities Council consolidated Grant No. ST/F012071/1 and continuation Grant No. ST/J000159/1, and the EU Seventh Framework through ENSAR(506065). K. T. F. was supported by STFC Advanced Fellowship Scheme Grant No. ST/G006415/1 and ERC Consolidator Grant No. 648381. We acknowledge the financial aid from the Ed Schneiderman Fund at New York University.

*ruben.degroote@fys.kuleuven.be

[1] S. R. Beane, E. Chang, S. Cohen, W. Detmold, H. W. Lin, K. Orginos, A. Parreño, M. J. Savage, and B. C. Tiburzi (NPLQCD Collaboration), Phys. Rev. Lett. 113, 252001 (2014).

[2] A. Ekström, G. R. Jansen, K. A. Wendt, G. Hagen, T. Papenbrock, B. D. Carlsson, C. Forssén, M. Hjorth-Jensen,
P. Navrátil, and W. Nazarewicz, Phys. Rev. C 91, 051301 (2015).

[3] Z.-T. Lu, P. Mueller, G. W. F. Drake, W. Nörtershäuser, S. C. Pieper, and Z.-C. Yan, Rev. Mod. Phys. 85, 1383 (2013).

[4] W. Nörtershäuser, C. Geppert, A. Krieger, K. Pachucki, M. Puchalski, K. Blaum, M. L. Bissell, N. Frömmgen, M. Hammen, M. Kowalska, J. Krämer, K. Kreim, R. Neugart, G. Neyens, R. Sánchez, and D. T. Yordanov, Phys. Rev. Lett. 115, 033002 (2015).

[5] J. Zhang, M. Tandecki, R. Collister, S. Aubin, J. A. Behr, E. Gomez, G. Gwinner, L. A. Orozco, M. R. Pearson, and G. D. Sprouse (FrPNC Collaboration), Phys. Rev. Lett. 115, 042501 (2015).

[6] K. Blaum, J. Dilling, and W. Nörtershäuser, Phys. Scr. T152, 014017 (2013).

[7] J. Papuga, M. L. Bissell, K. Kreim, C. Barbieri, K. Blaum, M. De Rydt, T. Duguet, R. F. Garcia Ruiz, H. Heylen, M. Kowalska, R. Neugart, G. Neyens, W. Nörtershäuser, M. M. Rajabali, R. Sánchez, N. Smirnova, V. Somà, and D. T. Yordanov, Phys. Rev. C 90, 034321 (2014).

[8] A. Nieminen, P. Campbell, J. Billowes, D. H. Forest, J. A. R. Griffith, J. Huikari, A. Jokinen, I. D. Moore, R. Moore, G. Tungate, and J. Äystö, Phys. Rev. Lett. 88, 094801 (2002).

[9] A. Voss, M. R. Pearson, J. Billowes, F. Buchinger, B. Cheal, J. E. Crawford, A. A. Kwiatkowski, C. D. P. Levy, and O. Shelbaya, Phys. Rev. Lett. 111, 122501 (2013).

[10] K. Minamisono, P. Mantica, A. Klose, S. Vinnikova, A. Schneider, B. Johnson, and B. Barquest, Nucl. Instrum. Methods Phys. Res., Sect. A 709, 85 (2013).

[11] H. Iimura, M. Koizumi, M. Miyabe, M. Oba, T. Shibata, N. Shinohara, Y. Ishida, T. Horiguchi, and H. A. Schuessler, Phys. Rev. C 68, 054328 (2003).

[12] K. Blaum, W. Geithner, J. Lassen, P. Lievens, K. Marinova, and R. Neugart, Nucl. Phys. A799, 30 (2008).

[13] B. Cheal et al., Phys. Rev. Lett. 104, 252502 (2010).

[14] V. N. Fedosseev, Y. Kudryavtsev, and V. I. Mishin, Phys. Scr. 85, 058104 (2012).

[15] H. D. Witte et al., Phys. Rev. Lett. 98, 112502 (2007).

[16] U. Köster, N. J. Stone, K. T. Flanagan, J. R. Stone, V. N. Fedosseev, K. L. Kratz, B. A. Marsh, T. Materna, L. Mathieu, P. L. Molkanov, M. D. Seliverstov, O. Serot, A. M. Sjödin, and Y. M. Volkov, Phys. Rev. C 84, 034320 (2011).

[17] R. Ferrer, N. Bree, T. Cocolios, I. Darby, H. D. Witte, W. Dexters, J. Diriken, J. Elseviers, S. Franchoo, M. Huyse, N. Kesteloot, Y. Kudryavtsev, D. Pauwels, D. Radulov, T. Roger, H. Savajols, P. V. Duppen, and M. Venhart, Phys. Lett. B 728, 191 (2014).

[18] Y. Kudryavtsev, R. Ferrer, M. Huyse, P. V. den Bergh, and P. V. Duppen, Nucl. Instrum. Methods Phys. Res., Sect. B 297, 7 (2013).

[19] Y. A. Kudriavtsev and V. S. Letokhov, Appl. Phys. B 29, 219 (1982).

[20] C. Schulz, E. Arnold, W. Borchers, W. Neu, R. Neugart, M. Neuroth, E. W. Otten, M. Scherf, K. Wendt, P. Lievens, Y. A. Kudryavtsev, V. S. Letokhov, V. I. Mishin, and V. V. Petrunin, J. Phys. B 24, 4831 (1991).

[21] K. T. Flanagan et al., Phys. Rev. Lett. 111, 212501 (2013).

[22] S. Rothe, V. N. Fedosseev, T. Kron, B. A. Marsh, R. E. Rossel, and K. D. A. Wendt, Nucl. Instrum. Methods Phys. Res., Sect. B 317, 561 (2013). 
[23] V. Meyer et al., Phys. Rev. Lett. 84, 1136 (2000).

[24] K. S. E. Eikema, W. Ubachs, W. Vassen, and W. Hogervorst, Phys. Rev. A 55, 1866 (1997).

[25] M. Hori and A. Dax, Opt. Lett. 34, 1273 (2009).

[26] M. Hori, A. Sótér, D. Barna, A. Dax, R. Hayano, S. Friedreich, B. Juhász, T. Pask, E. Widmann, D. Horváth et al., Nature (London) 475, 484 (2011).

[27] M. Reponen, I. Moore, T. Kessler, I. Pohjalainen, S. Rothe, and V. Sonnenschein, Eur. Phys. J. A 48, 45 (2012).

[28] J. Sell, K. Gulyuz, and G. Sprouse, Rev. Sci. Instrum. 80, 123108 (2009).

[29] N. Vitanov, B. Shore, L. Yatsenko, K. Böhmer, T. Halfmann, T. Rickes, and K. Bergmann, Opt. Commun. 199, 117 (2001).

[30] T. Halfmann, T. Rickes, N. Vitanov, and K. Bergmann, Opt. Commun. 220, 353 (2003).

[31] R. de Groote, G. Neyens, and K. Flanagan, Master's thesis, Leuven U. 2013.

[32] P. A. Butler, Rev. Mod. Phys. 68, 349 (1996).

[33] L. P. Gaffney et al., Nature (London) 497, 199 (2013).

[34] D. A. Fink et al., Phys. Rev. X 5, 011018 (2015).

[35] R. K. Sheline, Phys. Lett. B 197, 500 (1987).

[36] A. Coc, C. Thibault, F. Touchard, H. Duong, P. Juncar, S. Liberman, J. Pinard, J. Lermé, J. Vialle, S. Büttgenbach, A. Mueller, and A. Pesnelle, Phys. Lett. B 163, 66 (1985).
[37] H. T. Duong, P. Juncar, S. Liberman, A. C. Mueller, R. Neugart, E. W. Otten, B. Peuse, J. Pinard, H. H. Stroke, C. Thibault, F. Touchard, J. L. Vialle, K. Wendt (I. Collaboration), Europhys. Lett. 3, 175 (1987).

[38] I. Budinčevi et al., Phys. Rev. C 90, 014317 (2014).

[39] K. M. Lynch et al., Phys. Rev. X 4, 011055 (2014).

[40] G. Ewan, E. Hagberg, B. Jonson, S. Mattsson, and P. Tidemand-Petersson, Nucl. Phys. A380, 423 (1982).

[41] H. Frånberg, P. Delahaye, J. Billowes, K. Blaum, R. Catherall, F. Duval, O. Gianfrancesco, T. Giles, A. Jokinen, M. Lindroos, D. Lunney, E. Mane, and I. Podadera, Nucl. Instrum. Methods Phys. Res., Sect. B 266, 4502 (2008).

[42] T. Cocolios et al., Nucl. Instrum. Methods Phys. Res., Sect. B 317, 565 (2013).

[43] S. Aubin, E. Gomez, L. Orozco, and G. Sprouse, Phys. Rev. A 70, 042504 (2004).

[44] G. Neyens, Rep. Prog. Phys. 66, 1251 (2003).

[45] R. Casten, Nuclear Structure from a Simple Perspective (Oxford University Press, New York, 1990).

[46] I. Ragnarsson and S. G. Nilsson, Shapes and Shells in Nuclear Structure (Cambridge University Press, Cambridge, England, 2005).

[47] C. F. Liang, P. Paris, J. Kvasil, and R. K. Sheline, Phys. Rev. C 44, 676 (1991). 